Rektumkarzinom

\title{
Multimodales Therapiekonzept
}

Hochrisikopatienten mit Rektumkarzinom können von einer multimodalen Therapie profitieren. Darauf deuten die Ergebnisse einer Phase-II-Studie hin (Chua YJ et al., 2010, Lancet Oncol 11: 241-248).

In die einarmige Studie wurden 105 Patienten mit problematischen Rektumkarzinomen eingeschlossen, darunter Patienten mit T4-Tumoren, Patienten mit Lymphknotenmetastasen im Stadium N2 und Patienten mit T3-Tumoren in unmittelbarer Nachbarschaft zum Schließmuskel.

Den Ergebnisse der Studie zufolge könnten laut Werner Scheitauer, Wien/Österreich, mit einer Operation plus intensiver Radiochemotherapie auch bei diesen Patienten gute Langzeitergebnisse erzielt werden. Das multimodale Therapiekonzept in dieser Studie beinhaltete eine neoadjuvante Chemotherapie nach dem XELOX-Schema (Capecitabin/Oxaliplatin) sowie eine präoperative Bestrahlung. Sechs Wochen danach wurde eine totale Mastdarmexstirpation vorgenommen. Adjuvant erhielten die Patienten dann noch einmal drei Monate lang eine Chemotherapie mit Capecitabin.
89\% der Patienten konnten laut Scheitauer R0-reseziert werden. Das rezidivfreie Überleben nach drei Jahren lag bei $68 \%$ und das Gesamtüberleben bei 83\% (Tab.). Auf weitere Daten zur multimodalen Therapie bei Patienten mit Rektumkarzinom wird jetzt gewartet. Unter anderem wird in der aktuellen randomisierten Phase-III-Studie PETACC 6 (Pan European Trials in Adjuvant Colon Cancer) eine neoadjuvante Radiochemotherapie mit Capecitabin oder Capecitabin plus Oxaliplatin, gefolgt von der Operation und postoperativ jeweils sechs Zyklen Capecitabin oder Capecitabin plus Oxaliplatin untersucht. PG

Progressionsfreies (PFS) und Gesamtüberleben (OS)

\begin{tabular}{lll} 
& \multicolumn{3}{l}{ Intention-to-Treat-Gruppe $(\mathrm{n}=105)$} \\
& 3 Jahre [\%] (95-\%-KI) & 5 Jahre [\%] (95-\%-KI) \\
PFS & $68(59-77)$ & $64(55-74)$ \\
OS & $83(76-91)$ & $75(66-84)$
\end{tabular}

95-\%-Kl=95-\%-Konfidenzintervall nach Chua YJ et al., 2010, Lancet Oncol 11:241-248

\section{Rehabilitation in der Onkologie}

\section{Zunehmende Bedeutung in der Versorgung von Tumorpatienten}

Dass die Versorgung von Tumorpatienten mehr beinhaltet als Radio-/Chemotherapien und Tumoroperationen verdeutlichte Oliver Rick, Bad WildungenReinhardshausen. Rick informierte über aktuelle Entwicklungen aus dem Bereich der onkologischen Rehabilitation. Diese gewinnt im deutschen Gesundheitswesen zunehmend an Bedeutung: „Zwischen 2000 und 2008 stieg die Zahl der genehmigten Anträge von 135.000 auf $181.000^{\prime \prime}$, so Rick.

Der Experte warnte vor Vorurteilen bezüglich der onkologischen Rehabilitation. Die Deutsche Rentenversicherung, der wichtigste Träger von RehaLeistungen in der Onkologie, habe ermittelt, dass sich die Investitionen in eine Rehabilitation rein monetär bereits rechneten, wenn der Betreffende dadurch drei Monate eher ins Erwerbsleben zurückkehren könne.

Zu den evidenzbasierten Pfeilern der onkologischen Rehabilitation zählt Rick die Sport- und Bewegungstherapie, die Ernährungstherapie und die Psychoonkologie. So sei regelmäßige körperliche Aktivität bei Mammakarzinompatientinnen in der Nurses Health Study mit einem deutlich besseren Gesamtüberleben und einer deutlich geringeren tumorspezifischen Mortalität assoziiert gewesen. „Ähnliche Daten gibt es auch für Patienten mit kolorektalem Karzinom", so Rick.

Als aktuelles Beispiel für eine randomisierte, kontrollierte Studie im Bereich der onkologischen Rehabilitation nannte Rick eine Untersuchung zum Effekt eines zweimal wöchentlichen Krafttrainings der Arme auf die Symp- tome bei Patientinnen mit sekundärem Lymphödem nach Mammakarzinomoperation (Schmitz KH et al., 2009, N Engl J Med 361: 664-673). Insgesamt nahmen 141 Patientinnen an der Studie teil. In der Interventions- und in der Kontrollgruppe wurde unabhängig vom Krafttraining eine Kompressionstherapie durchgeführt.

Es zeigte sich ein positiver Effekt des Krafttrainings: Bei 14\% der Patientinnen wurde eine Exazerbation des Lymphödems beobachtet verglichen mit einem Anteil von 29\% im Kontrollarm. Auch die Beschwerden waren mit dem Krafttraining geringer als ohne. Beim primären Endpunkt, dem Anteil der Patientinnen mit einer Schwellung von Arm und Hand $\geq 5 \%$, zeigte sich in dieser Studie allerdings kein Unterschied. PG 